\title{
Remarks on Weakly KKM Maps in Abstract Convex Spaces
}

\author{
Sehie Park ${ }^{1,2}$ \\ ${ }^{1}$ Natural Sciences Division, The National Academy of Sciences, Seoul 137-044, South Korea \\ ${ }^{2}$ Department of Mathematics, Seoul National University, Seoul 151-747, South Korea
}

Correspondence should be addressed to Sehie Park, shpark@math.snu.ac.kr

Received 26 September 2007; Accepted 7 January 2008

Recommended by Petru Jebelean

A KKM space is an abstract convex space satisfying the KKM principle. We obtain variants of the KKM principle for KKM spaces related to weakly KKM maps and indicate some applications of them. These results properly generalize the corresponding ones in G-convex spaces and $\phi_{A}$-spaces $\left(X, D ;\left\{\phi_{A}\right\}_{A \in\langle D\rangle}\right)$. Consequently, results by Balaj 2004, Liu 1991, and Tang et al. 2007 can be properly generalized and unified.

Copyright (C) 2008 Sehie Park. This is an open access article distributed under the Creative Commons Attribution License, which permits unrestricted use, distribution, and reproduction in any medium, provided the original work is properly cited.

\section{Introduction}

Since the appearance of generalized convex (simply, G-convex) spaces in 1993 [1], the concept has been challenged by several authors who aimed to obtain more general concepts. In fact, a number of modifications or imitations of the concept have followed, for example, $L$-spaces due to Ben-El-Mechaiekh et al. [2], spaces having property $(H)$ due to Huang [3], FC-spaces due to Ding $[4,5]$, and others. It is known that all of such examples belong to the class of $\phi_{A}$-spaces and are particular forms of G-convex spaces; see [6]. Some authors also tried to generalize the Knaster-Kuratowski-Mazurkiewicz theorem (simply, the KKM principle) [7] for their own setting. They introduced various types of generalized KKM maps and obtained modifications of known results. Recently, we proposed new concepts of abstract convex spaces and KKM spaces [8-11] which are proper generalizations of G-convex spaces.

In 1991, Liu [12] obtained a form of the KKM principle and applied it to SupInfSup inequalities of von Neumann type and of Ky Fan type. Motivated by this work, Balaj [13] introduced the concept of weakly G-KKM mappings for G-convex spaces and obtained related results on intersections and the Fan type or the Sion type minimax inequalities. Moreover, based 
on the misconception that FC-spaces generalize G-convex spaces, Tang et al. [14] introduced the so-called W-G-F-KKM mapping and claimed to obtain similar results for the so-called FCspaces.

In the present paper, our aim is to show that such basic results for weakly KKM maps on G-convex spaces can be extended on the more general KKM spaces [6, 10,11], a particular type of abstract convex spaces satisfying the general KKM principle. These results properly generalize the corresponding ones in $G$-convex spaces and $\phi_{A}$-spaces $\left(X, D ;\left\{\phi_{A}\right\}_{A \in\langle D\rangle}\right)$. We note that some applications of them in [12-14] can also be generalized to KKM spaces. Consequently, most results in [12-14] can be properly generalized and unified.

\section{Abstract convex spaces}

In this section, we follow mainly $[6,8,9,15]$.

Let $\langle D\rangle$ denote the set of all nonempty finite subsets of a set $D$.

Definition 2.1. An abstract convex space $(E, D ; \Gamma)$ consists of a set $E$, a nonempty set $D$, and a multimap $\Gamma:\langle D\rangle \multimap E$ with nonempty values. One may denote $\Gamma_{A}:=\Gamma(A)$ for $A \in\langle D\rangle$.

For any $D^{\prime} \subset D$, the $\Gamma$-convex hull of $D^{\prime}$ is denoted and defined by

$$
\operatorname{co}_{\Gamma} D^{\prime}:=\bigcup\left\{\Gamma_{A} \mid A \in\left\langle D^{\prime}\right\rangle\right\}
$$

(co is reserved for the convex hull in vector spaces).

A subset $X$ of $E$ is called a $\Gamma$-convex subset of $(E, D ; \Gamma)$ relative to $D^{\prime}$ if for any $N \in\left\langle D^{\prime}\right\rangle$, one has $\Gamma_{N} \subset X$, that is, $\mathrm{co}_{\Gamma} D^{\prime} \subset X$. This means that $\left(X, D^{\prime} ;\left.\Gamma\right|_{\left\langle D^{\prime}\right\rangle}\right)$ itself is an abstract convex space called a subspace of $(E, D ; \Gamma)$.

When $D \subset E$, the space is denoted by $(E \supset D ; \Gamma)$. In such case, a subset $X$ of $E$ is said to be $\Gamma$-convex if, for any $A \in\langle X \cap D\rangle$, one has $\Gamma_{A} \subset X$. In case $E=D$, let $(E ; \Gamma):=(E, E ; \Gamma)$.

Example 2.2. In $[6,16]$, we gave plenty of examples of abstract convex spaces. Here we give only two of them as follows.

(1) Usually, a convexity space $(E, \mathcal{C})$ in the classical sense consists of a nonempty set $E$ and a family $\mathcal{C}$ of subsets of $E$ such that $E$ itself is an element of $\mathcal{C}$ and $\mathcal{C}$ is closed under arbitrary intersection. For details, see [17], where the bibliography lists 283 papers. For any subset $X \subset E$, its $\mathcal{C}$-convex hull is defined and denoted by $\operatorname{Co}_{\mathcal{C}} X:=\bigcap\{Y \in \mathcal{C} \mid X \subset Y\}$. We say that $X$ is $\mathcal{C}$ convex if $X=\operatorname{Coc}_{C} X$. Now we can consider the map $\Gamma:\langle E\rangle \multimap E$ given by $\Gamma_{A}:=\operatorname{Co}_{C} A$. Then $(E, \mathcal{C})$ becomes our abstract convex space $(E ; \Gamma)$.

(2) A generalized convex space or a G-convex space $(E, D ; \Gamma)$ consists of a topological space $E$, a nonempty set $D$, and a multimap $\Gamma:\langle D\rangle \multimap E$ such that for each $A \in\langle D\rangle$ with the cardinality $|A|=n+1$, there exists a continuous function $\phi_{A}: \Delta_{n} \rightarrow \Gamma(A)$ such that $J \in\langle A\rangle$ implies $\phi_{A}\left(\Delta_{J}\right) \subset \Gamma(J)$.

Here, $\Delta_{n}$ is a standard $n$-simplex with vertices $\left\{e_{i}\right\}_{i=0}^{n}$, and $\Delta_{J}$ the face of $\Delta_{n}$ corresponding to $J \in\langle A\rangle$; that is, if $A=\left\{a_{0}, a_{1}, \ldots, a_{n}\right\}$ and $J=\left\{a_{i_{0}}, a_{i_{1}}, \ldots, a_{i_{k}}\right\} \subset A$, then $\Delta_{J}=\operatorname{co}\left\{e_{i_{0}}, e_{i_{1}}, \ldots, e_{i_{k}}\right\}$.

We have established a large amount of literature on G-convex spaces; see [1, 16, 18-21] and references therein.

Recently, we are concerned with another variant of G-convex spaces as follows [6]. 
Definition 2.3. A $\phi_{A}$-space

$$
\left(X, D ;\left\{\phi_{A}\right\}_{A \in\langle D\rangle}\right)
$$

consists of a topological space $X$, a nonempty set $D$, and a family of continuous functions $\phi_{A}: \Delta_{n} \rightarrow X$ (i.e., singular $n$-simplexes) for $A \in\langle D\rangle$ with the cardinality $|A|=n+1$.

Example 2.4. The following are typical examples of $G$-convex spaces and $\phi_{A}$-spaces:

(1) any nonempty convex subset of a topological vector space (t.v.s.);

(2) [22] a convex space due to Komiya;

(3) [23] a convex space due to Lassonde;

(4) $[24,25]$ a $C$-space (or an $H$-space) due to Horvath;

(5) [2] an $L$-space due to Ben-El-Mechaiekh et al.;

(6) [3] a topological space $Y$ is said to have property $(H)$ if, for each $N=\left\{y_{0}, \ldots, y_{n}\right\} \in\langle Y\rangle$, there exists a continuous mapping $\varphi_{N}: \Delta_{n} \rightarrow Y$;

(7) $[4,5,26,27]\left(Y,\left\{\varphi_{N}\right\}\right)$ is said to be an FC-space if $Y$ is a topological space and for each $N=\left\{y_{0}, \ldots, y_{n}\right\} \in\langle Y\rangle$, where some elements in $N$ may be the same, there exists a continuous mapping $\varphi_{N}: \Delta_{n} \rightarrow Y$;

(8) any $G$-convex space is clearly a $\phi_{A}$-space. The converse also holds.

Proposition 2.5 (see [6]). A $\phi_{A}$-space $\left(X, D ;\left\{\phi_{A}\right\}_{A \in\langle D\rangle}\right)$ can be made into a G-convex space $(X, D ; \Gamma)$.

Proof. This can be done in two ways.

(1) For each $A \in\langle D\rangle$, by putting $\Gamma_{A}:=X$, we obtain a trivial G-convex space $(X, D ; \Gamma)$.

(2) Let $\left\{\Gamma^{\alpha}\right\}_{\alpha}$ be the family of maps $\Gamma^{\alpha}:\langle D\rangle \multimap X$ giving a G-convex space $\left(X, D ; \Gamma^{\alpha}\right)$ such that $\phi_{A}\left(\Delta_{n}\right) \subset \Gamma_{A}^{\alpha}$ for each $A \in\langle D\rangle$ with $|A|=n+1$. Note that, by (1), this family is not empty. Then, for each $\alpha$ and each $A \in\langle D\rangle$ with $|A|=n+1$, we have

$$
\phi_{A}\left(\Delta_{n}\right) \subset \Gamma_{A^{\prime}}^{\alpha} \quad \phi_{A}\left(\Delta_{J}\right) \subset \Gamma_{J}^{\alpha} \quad \text { for } J \subset A .
$$

Let $\Gamma:=\bigcap_{\alpha} \Gamma^{\alpha}$, that is, $\Gamma_{A}=\bigcap_{\alpha} \Gamma_{A}^{\alpha}$. Then

$$
\phi_{A}\left(\Delta_{n}\right) \subset \Gamma_{A}, \quad \phi_{A}\left(\Delta_{J}\right) \subset \Gamma_{J} \quad \text { for } J \subset A .
$$

Therefore, $(X, D ; \Gamma)$ is a $G$-convex space.

Therefore, $G$-convex spaces and $\phi_{A}$-spaces are essentially the same.

For a $G$-convex space $(X, D ; \Gamma)$, a multimap $G: D \multimap X$ is called a $K K M$ map if $\Gamma_{A} \subset G(A)$ for each $A \in\langle D\rangle$.

Proposition 2.6 (see [6]). For a $\phi_{A}$-space $\left(X, D ;\left\{\phi_{A}\right\}_{A \in\langle D\rangle}\right)$, any map $T: D \multimap X$ satisfying

$$
\phi_{A}\left(\Delta_{J}\right) \subset T(J) \text { for each } A \in\langle D\rangle, J \in\langle A\rangle,
$$

is a KKM map on a G-convex space $(X, D ; \Gamma)$. 
Proof. Define $\Gamma:\langle D\rangle \multimap X$ by $\Gamma_{A}:=T(A)$ for each $A \in\langle D\rangle$. Then $(X, D ; \Gamma)$ becomes a $G$-convex space. In fact, for each $A$ with $|A|=n+1$, we have a continuous function $\phi_{A}: \Delta_{n} \rightarrow T(A)=$ : $\Gamma(A)$ such that $J \in\langle A\rangle$ implies $\phi_{A}\left(\Delta_{J}\right) \subset T(J)=: \Gamma(J)$. Moreover, note that $\Gamma_{A} \subset T(A)$ for each $A \in\langle D\rangle$ and hence $T: D \multimap X$ is a KKM map on a $G$-convex space $(X, D ; \Gamma)$.

Remark 2.7. In [14], its authors repeated Ding's false claim in a large number of his own papers as follows. "Recently, Ding [4] introduced FC-space which extended G-convex space further and proved the corresponding KKM theorem. From this, many new KKM-type theorems and applications were founded in FC-spaces." For Ding's claim, see $[5,26,27]$ and references of [6]. One wonders how a pair $\left(X,\left\{\varphi_{A}\right\}\right)$ could extend the triple $(X, D ; \Gamma)$.

The concept of KKM maps for G-convex spaces is refined as follows.

Definition 2.8. Let $(E, D ; \Gamma)$ be an abstract convex space and $Z$ a set. For a multimap $F: E \multimap Z$ with nonempty values, if a multimap $G: D \multimap Z$ satisfies

$$
F\left(\Gamma_{A}\right) \subset G(A):=\bigcup_{y \in A} G(y) \quad \forall A \in\langle D\rangle,
$$

then $G$ is called a KKM map with respect to $F$. A KKM map $G: D \multimap E$ is a KKM map with respect to the identity map $1_{E}$.

A multimap $F: E \multimap Z$ is said to have the KKM property and called $a \mathfrak{K}$-map if, for any KKM map $G: D \multimap Z$ with respect to $F$, the family $\{G(y)\}_{y \in D}$ has the finite intersection property. We denote

$$
\mathfrak{K}(E, Z):=\{F: E \multimap Z \mid F \text { is a } \mathfrak{K} \text {-map }\} \text {. }
$$

Similarly, when $Z$ is a topological space, a $\Re \mathfrak{R}$-map is defined for closed-valued maps $G$, and a $\mathfrak{R O}$-map for open-valued maps $G$. Note that if $Z$ is discrete, then three classes $\mathfrak{K}, \mathfrak{R C}$, and $\mathfrak{R O}$ are identical. Some authors use the notation $\operatorname{KKM}(E, Z)$ instead of $\mathfrak{R C}(E, Z)$.

Example 2.9. The above terminology unifies various concepts in other author's usage as follows.

(1) Every abstract convex space in our sense has a map $F \in \mathfrak{K}(E, Z)$ for any nonempty set $Z$. In fact, for each $x \in E$, choose $F(x):=Z$ or $F(x):=\left\{z_{0}\right\}$ for some $z_{0} \in Z$.

If $1_{E} \in \mathfrak{K}(E, E)$, then $f \in \mathfrak{K}(E, Z)$ for any function $f: E \rightarrow Z$. If $E$ and $Z$ have any topology, this holds for $\mathfrak{R C}$ or $\mathfrak{R O}$ for any continuous $f$.

(2) For a $G$-convex space $(X, D ; \Gamma)$ and a topological space $Z$, we defined the classes $\mathfrak{K}, \mathfrak{R C}, \mathfrak{R O}$ of multimaps $F: X \multimap Z[16]$. It is known that for a $G$-convex space $(X, D ; \Gamma)$, we have the identity map $1_{X} \in \mathfrak{R C}(X, X) \cap \mathfrak{R O}(X, X)$; see [19-21]. Moreover, for any topological space $Y$, if $F: X \rightarrow Y$ is a continuous single-valued map or if $F: X \multimap Y$ has a continuous selection, then $F \in \mathfrak{R C}(X, Y) \cap \mathfrak{R O}(X, Y)$.

(3) Let $(X, D ; \Gamma)$ be a G-convex space, $Y$ a nonempty set, and $T: X \rightarrow 2^{Y}, S: D \rightarrow 2^{Y}$ two mappings. We say that $S$ is a generalized G-KKM mapping [13] with respect to $T$ if for each $A \in\langle D\rangle, T(\Gamma(A)) \subset S(A)$. If $Y$ is a topological space, $T: X \rightarrow 2^{Y}$ is said to have the G$K K M$ property if for any map $S: D \rightarrow 2^{Y}$ generalized G-KKM with respect to $T$, the family $\{\overline{S(z)} \mid z \in D\}$ has the finite intersection property. 
This simply tells that $S$ is a KKM map with respect to $T$ and $T \in \mathfrak{R C}(X, Y)$.

(4) Let $\left(X, \varphi_{A}\right)$ be an FC-space, $Y$ a nonempty set, and $T, S: X \rightarrow 2^{Y}$ two mappings. We say that $S$ is a generalized KKM mapping [14] with respect to $T$ if for each $A \in\langle X\rangle$, each $B \in\langle A\rangle$, $T\left(\varphi_{A}\left(\Delta_{B}\right)\right) \subset S(B)$. If $Y$ is a topological space, $T: X \rightarrow 2^{Y}$ is said to have the F-KKM property if for any map $S: X \rightarrow 2^{Y}$ generalized KKM with respect to $T$, the family $\{\overline{S(z)} \mid z \in X\}$ has the finite intersection property.

Note that $\left(X, \varphi_{A}\right)$ becomes a $G$-convex space $(X, A ; \Gamma)$ for each $A \in\langle X\rangle$ with $\Gamma(B):=$ $\varphi_{A}\left(\Delta_{B}\right)$ for each $B \in\langle A\rangle$. Then $\left.S\right|_{A}: A \rightarrow 2^{Y}$ is a KKM map with respect to $T: X \rightarrow 2^{Y}$ and $T \in \mathfrak{R C}(X, Y)$.

(5) Let $\left(X, \varphi_{A}\right)$ be an FC-space, $Y$ a nonempty subset, and $S: Y \rightarrow 2^{X}$. Then $S$ is a generalized F-KKM mapping [14] if for each finite subset $\widetilde{A}=\left\{y_{0}, \ldots, y_{n}\right\}$ of $Y$, there exists a finite subset $A=\left\{x_{0}, \ldots, x_{n}\right\}$ of $X$ such that for any subset $B=\left\{x_{i_{0}}, \ldots, x_{i_{k}}\right\}$ of $A$,

$$
\varphi_{A}\left(\operatorname{co}\left\{e_{i_{0}}, \ldots, e_{i_{k}}\right\}\right) \subset \bigcup_{j=0}^{k} S\left(y_{i_{j}}\right) .
$$

A generalized F-KKM map in the above sense can be made into a KKM map on a Gconvex space $(X \supset Y ; \Gamma)$ where $\Gamma(\tilde{A}):=S(\tilde{A})$ and $\phi_{\tilde{A}}:=\varphi_{A}$ as above.

\section{The KKM spaces}

We introduced the following in [6].

Definition 3.1. For an abstract convex topological space $(E, D ; \Gamma)$, the KKM principle is the statement $1_{E} \in \mathfrak{R C}(E, E) \cap \mathfrak{R O}(E, E)$.

A KKM space is an abstract convex topological space satisfying the KKM principle.

In our recent work [9], we studied elements or foundations of the KKM theory on abstract convex spaces and noticed that many important results therein are related to KKM spaces. Moreover, in [10,11], a fundamental theory and its applications on KKM spaces are extensively investigated.

Example 3.2. We give examples of KKM spaces.

(1) Every G-convex space is a KKM space; see [19-21].

(2) A connected ordered space $(X, \leq)$ can be made into an abstract convex topological space $(X \supset D ; \Gamma)$ for any nonempty $D \subset X$ by defining $\Gamma_{A}:=[\min A, \max A]=\{x \in X \mid$ $\min A \leq x \leq \max A\}$ for each $A \in\langle D\rangle$. Further, it is a KKM space; see [15, Theorem 5(i)].

(3) The extended long line $L^{*}$ can be made into a KKM space $\left(L^{*} \supset D ; \Gamma\right)$; see [15]. In fact, $L^{*}$ is constructed from the ordinal space $D:=[0, \Omega]$ consisting of all ordinal numbers less than or equal to the first uncountable ordinal $\Omega$, together with the order topology. Recall that $L^{*}$ is a generalized arc obtained from $[0, \Omega]$ by placing a copy of the interval $(0,1)$ between each ordinal $\alpha$ and its successor $\alpha+1$ and we give $L^{*}$ the order topology. Now let $\Gamma:\langle D\rangle \multimap L^{*}$ be the one as in (2).

But $L^{*}$ is not a $G$-convex space. In fact, since $\Gamma\{0, \Omega\}=L^{*}$ is not path connected, for $A:=\{0, \Omega\} \in\left\langle L^{*}\right\rangle$ and $\Delta_{1}:=[0,1]$, there does not exist a continuous function $\phi_{A}:[0,1] \rightarrow \Gamma_{A}$ such that $\phi_{A}\{0\} \subset \Gamma\{0\}=\{0\}$ and $\phi_{A}\{1\} \subset \Gamma\{\Omega\}=\{\Omega\}$. Therefore, $\left(L^{*} \supset D ; \Gamma\right)$ is not G-convex. 
Therefore, the concepts of KKM spaces properly generalize those of G-convex spaces and $\phi_{A}$-spaces.

From the definition of the KKM map, we have the following form of Fan's matching theorem.

Theorem 3.3. Let $(E, D ; \Gamma)$ be a KKM space and $S: D \multimap E$ a map satisfying what follows:

(1) $S(z)$ is open (resp., closed) for each $z \in D$;

(2) $X=\bigcup_{z \in M} S(z)$ for some $M \in\langle D\rangle$.

Then there exists an $N \in\langle D\rangle$ such that

$$
\Gamma_{N} \cap \bigcap_{z \in N} S(z) \neq \varnothing .
$$

Proof. Let $G: D \multimap E$ be a map given by $G(z):=E \backslash S(z)$ for $z \in D$. Then $G$ has closed (resp., open) values. Suppose, on the contrary to the conclusion, that for any $N \in\langle D\rangle$, we have $\Gamma_{N} \cap \bigcap_{z \in N} S(z)=\varnothing$, that is, $\Gamma_{N} \subset E \backslash \bigcap_{z \in N} S(z)=\bigcup_{z \in N}(E \backslash S(z))=G(N)$. Therefore, $G$ is a KKM map. Since $(E, D ; \Gamma)$ is a KKM space, there exists a $\widehat{y} \in \bigcap_{z \in M} G(z)=\bigcap_{z \in M}(X \backslash S(z))$. Hence, $\widehat{y} \notin S(z)$ for all $z \in M$. This violates condition (2).

Corollary 3.4. Let $(E, D ; \Gamma)$ be a KKM space, $A \in\langle D\rangle,\left\{M_{z} \mid z \in A\right\}$ an open or closed cover of $E$. Then there exists a nonempty subset $B$ of $A$ such that $\Gamma(B) \cap \cap\left\{M_{z} \mid z \in B\right\} \neq \varnothing$.

Corollary 3.5 (see [13, Lemma 1]). Let $(X, D ; \Gamma)$ be a G-convex space, $A \in\langle D\rangle,\left\{M_{z} \mid z \in A\right\}$ an open or closed cover of $X$. Then there exists a nonempty subset $B$ of $A$ such that $\Gamma(B) \cap \cap\left\{M_{z} \mid z \in\right.$ $B\} \neq \varnothing$.

Balaj [13] deduced Corollary 3.5 from a previous result of the present author.

Corollary 3.6 (see [14, Theorem 3.2]). Let $\left(X, \varphi_{A}\right)$ be an FC-space, $A \in\langle X\rangle,\left\{M_{x} \mid x \in A\right\}$ an open or closed cover of $X$. Then there exists a nonempty subset $B$ of $A$ such that $\varphi_{A}\left(\Delta_{B}\right) \cap \cap\left\{M_{x} \mid\right.$ $x \in B\} \neq \varnothing$.

This is a very particular form of Corollary 3.5 with $\Gamma(B):=\varphi_{A}\left(\Delta_{B}\right)$. In fact, $\left(X, \varphi_{A}\right)$ becomes a $G$-convex space $(X, A ; \Gamma)$ with $\Gamma(B):=\varphi_{A}\left(\Delta_{B}\right)$ for each $A \in\langle X\rangle$ and each $B \in\langle A\rangle$.

Note also that our proof of Theorem 3.3 is much more simple than that of [14, Theorem 3.2].

\section{Weakly KKM maps}

Definition 4.1. Let $(E, D ; \Gamma)$ be an abstract convex space and $Z$ a set. For a multimap $F: E \multimap Z$ with nonempty values, if a multimap $G: D \multimap Z$ satisfies

$$
F(x) \cap G(A) \neq \varnothing \quad \forall A \in\langle D\rangle \text { and all } x \in \Gamma(A),
$$

then $G$ is called a weakly KKM map with respect to $F$.

Clearly, each KKM map with respect to $F$ is weakly KKM, and a weakly KKM map $G: D \multimap E$ with respect to the identity map $1_{E}$ is simply a KKM map. 
Example 4.2. (1) When $X:=E$ is a nonempty subset of a vector space, $F$ is said to be trappable by $G^{c}$ iff $G$ is not weakly KKM with respect to $F$, where $G^{c}(a):=Z \backslash G(a)$ for each $a \in D$ [12].

(2) Let $(X, D ; \Gamma)$ be a $G$-convex space, $Y$ a nonempty set, and $T: X \rightarrow 2^{Y}, S: D \rightarrow 2^{Y}$ two mappings [13]. We say that $S$ is weakly G-KKM mapping with respect to $T$ if for each $A \in\langle D\rangle$ and any $x \in \Gamma(A), T(x) \cap S(A) \neq \varnothing$.

(3) Let $\left(X, \varphi_{A}\right)$ be an FC-space, $Y$ a nonempty set, and $T, S: X \rightarrow 2^{Y}$ two mappings [14]. We say that $S$ is weakly generalized F-KKM mapping with respect to $T$ (for short, W-G-F-KKM mapping with respect to $T)$ if for each $A \in\langle X\rangle$, each $B \subset A$ and any $x \in \varphi_{A}\left(\Delta_{B}\right), T(x) \cap S(B) \neq \varnothing$.

In 1991, Liu [12] obtained a form of the KKM principle. Motivated by the form, we deduce the following generalization.

Theorem 4.3. Let $(X, D ; \Gamma)$ be a compact KKM space, $Y$ a nonempty set, and $F: X \multimap Y$ and $G$ : $D \multimap Y$ maps such that

(1) $G$ is weakly KKM map with respect to $F$

(2) for each $z \in D$, the set $\{x \in X \mid F(x) \cap G(z) \neq \varnothing\}$ is closed.

Then there exists an $x_{0} \in X$ such that $F\left(x_{0}\right) \cap G(z) \neq \varnothing$ for each $z \in D$.

Proof. Suppose that the conclusion does not hold. Then for each $x \in X$, there exists a $z \in D$ such that $F(x) \cap G(z)=\varnothing$. Define an open set

$$
M_{z}:=\{x \in X \mid F(x) \cap G(z)=\varnothing\} \text { for } z \in D
$$

Since $X$ is compact, there is an $A \in\langle D\rangle$ such that $\left\{M_{z} \mid z \in A\right\}$ is an open cover of $X$. Then, by Corollary 3.4, there exist a subset $B$ of $A$ and a point

$$
x_{0} \in \Gamma(B) \cap \bigcap\left\{M_{z} \mid z \in B\right\} \neq \varnothing .
$$

Since $G$ is weakly KKM with respect to $F$ and $x_{0} \in \Gamma(B)$, we have $F\left(x_{0}\right) \cap G(B) \neq \varnothing$.

On the other hand, $x_{0} \in \bigcap\left\{M_{z} \mid z \in B\right\}$ implies $F\left(x_{0}\right) \cap G(z)=\varnothing$ for all $z \in B$ and hence, $F\left(x_{0}\right) \cap G(B)=\varnothing$. This is a contradiction.

Corollary 4.4 (see $[13$, Theorem 2]). Let $(X, D ; \Gamma)$ be a compact G-convex space, $Y$ a nonempty set, and $T: X \multimap Y$ and $S: D \multimap Y$ two maps such that

(i) $S$ is weakly G-KKM map with respect to $T$;

(ii) for each $z \in D$, the set $\{x \in X \mid T(x) \cap S(z) \neq \varnothing\}$ is closed.

Then there exists an $x_{0} \in X$ such that $T\left(x_{0}\right) \cap S(z) \neq \varnothing$ for each $z \in D$.

The following is an immediate consequence of Corollary 4.4.

Corollary 4.5 (see [14, Theorem 3.3]). Let $\left(X, \varphi_{A}\right)$ be a compact FC-space, $Y$ a nonempty set, and $T, S: X \multimap Y$ two maps such that 
(i) $S$ is $W-G-F-K K M$ map with respect to $T$;

(ii) for each $z \in D$, the set $\{x \in X \mid T(x) \cap S(z) \neq \varnothing\}$ is closed.

Then there exists an $x_{0} \in X$ such that $T\left(x_{0}\right) \cap S(z) \neq \varnothing$ for each $z \in D$.

Example 4.6. In [13, Remark 1], it is noted that condition (2) in Theorem 4.3 is satisfied if $Y$ is a topological space, $F$ is upper semicontinuous, and $G$ has closed values. In this case, [12, Theorem 2.1] is for a compact convex subset $X=D$ of a topological vector space, and [14, Theorem 3.4] for a compact $F C$-space $\left(X, \varphi_{A}\right)$.

We have the following variant of Theorem 4.3.

Theorem 4.7. Let $(X, D ; \Gamma)$ be a KKM space, $Y$ a nonempty set, and $F: X \multimap Y$ and $G: D \multimap Y$ maps such that

(1) $G$ is weakly KKM with respect to $F$;

(2) the set $\{x \in X \mid F(x) \cap G(z) \neq \varnothing\}$ is either all closed or all open for all $z \in D$.

Then for each $A \in\langle D\rangle$ there exists an $x_{0} \in \Gamma(A)$ such that $F\left(x_{0}\right) \cap G(z) \neq \varnothing$ for all $z \in A$.

Proof. Let $A \in\langle D\rangle$. Then it is easily checked that the subspace $\left(X, A ;\left.\Gamma\right|_{\langle A\rangle}\right)$ is also a KKM space. Now the conclusion follows from the same argument in the proof of Theorem 4.3.

Corollary 4.8 (see [13, Theorem 3]). Let $(X, D ; \Gamma)$ be a G-convex space, $Y$ a nonempty set, and $T: X \multimap Y$ and $S: D \multimap Y$ maps such that

(i) $S$ is weakly G-KKM map with respect to $T$;

(ii) the set $\{x \in X \mid T(x) \cap S(z) \neq \varnothing\}$ is either all closed or all open for all $z \in D$.

Then for each $A \in\langle D\rangle$ there exists an $x_{0} \in \Gamma(A)$ such that $T\left(x_{0}\right) \cap S(z) \neq \varnothing$ for all $z \in A$.

From Corollary 4.8, we have the following.

Corollary 4.9 (see [14, Theorem 3.5]). Let $\left(X, \varphi_{A}\right)$ be an FC-space, $\varphi_{A}\left(\Delta_{n}\right)$ an FC-subspace for each $A \in\langle X\rangle, Y$ a nonempty set, and $T, S: X \multimap Y$ two maps such that

(i) $S$ is $W-G-F-K K M$ map with respect to $T$;

(ii) the set $\{x \in X \mid T(x) \cap S(z) \neq \varnothing\}$ is either all closed or all open for all $z \in D$.

Then for each $A \in\langle X\rangle$ there exists an $x_{0} \in \varphi_{A}\left(\Delta_{n}\right)$ such that $T\left(x_{0}\right) \cap S(z) \neq \varnothing$ for each $z \in \varphi_{A}\left(\Delta_{n}\right)$.

Remark 4.10. In [13, Remark 2], it is noted that condition (3.2) in Theorem 4.7 is satisfied if $Y$ is a topological space and either $F$ is upper semicontinuous and $G$ has closed values or $F$ is lower semicontinuous and $G$ has open values. This is exploited in [14, Theorem 3.6].

As an example of applications of Theorem 4.3, we give the following.

Theorem 4.11. Let $(X, D ; \Gamma)$ be a compact KKM space, $Y$ a topological space. Let $T: X \multimap Y$ be an upper semicontinuous map, $\psi: D \times Y \rightarrow \mathbb{R}, \varphi: X \times Y \rightarrow \mathbb{R}$ two functions and $\beta=$ $\inf _{x \in X} \sup _{y \in T(x)} \varphi(x, y)$. Suppose that 
(1) for each $z \in D, \psi(z, \cdot)$ is upper semicontinuous on $Y$;

(2) for any $\lambda<\beta$ and $y \in T(x), c_{\Gamma}\{z \in D \mid \psi(z, y)<\lambda\} \subset\{x \in X \mid \varphi(x, y)<\lambda\}$.

(a) Then the following holds:

$$
\inf _{x \in X} \sup _{y \in T(x)} \varphi(x, y) \leq \sup _{x \in X} \inf \sup _{z \in D} \psi(z, y)
$$

(b) Further, if $T$ is compact valued, then there exists an $x_{0} \in X$ such that

$$
\inf _{x \in X} \sup _{y \in T(x)} \varphi(x, y) \leq \inf _{z \in D} \sup _{y \in T\left(x_{0}\right)} \psi(z, y) .
$$

Proof. Just follow that of [13, Theorem 4].

Corollary 4.12. In Theorem 4.11, $(X, D ; \Gamma)$ can be replaced by a compact G-convex space without affecting its conclusion.

Note that Corollary 4.12 contains some known forms of the Fan type minimax inequalities; see [13].

Corollary 4.13 (see [14, Theorem 4.1]). Let $\left(X, \varphi_{A}\right)$ be a compact FC-space and $Y$ a topological space. Let $T: X \multimap Y$ be a u.s.c. map, $f, g: X \times Y \rightarrow \mathbb{R}$ two functions, and $\beta=\inf _{x \in X} \sup _{y \in T(x)} f(x, y)$. Suppose that

(i) for each $z \in X, g(z, \cdot)$ is u.s.c. on $Y$;

(ii) for any $\lambda<\beta$ and $y \in T(x)$, if for each $A \in\langle X\rangle$ and $B \in\langle A \cap\{x \in X \mid g(x, y)<\lambda\}\rangle$ one has $\varphi_{A}\left(\Delta_{B}\right) \subset\{x \in X \mid f(x, y)<\lambda\}$.

(a) Then the following holds:

$$
\inf _{x \in X} \sup _{y \in T(x)} f(x, y) \leq \sup _{x \in X} \inf \sup _{z \in X} g(z, y)
$$

(b) Moreover, if $T$ is compact-valued, then there exists an $x_{0} \in X$ such that

$$
\inf _{x \in X} \sup _{y \in T(x)} f(x, y) \leq \inf _{z \in X} \sup _{y \in T\left(x_{0}\right)} g(z, y)
$$

\section{Further remarks}

Until now, in this paper, we showed that basic results in [12] for topological vector spaces, in [13] for G-convex spaces, and in [14] for FC-spaces, are all extended to KKM spaces. Therefore, most of their applications in each paper can be also generalized to KKM spaces. The readers can show this in case they are urgently needed. Finally, note that results in [14] are all particular to corresponding ones for G-convex spaces. 


\section{References}

[1] S. Park and H. Kim, "Admissible classes of multifunctions on generalized convex spaces," Proceedings of College of Natural Sciences, Seoul National University, vol. 18, pp. 1-21, 1993.

[2] H. Ben-El-Mechaiekh, S. Chebbi, M. Florenzano, and J.-V. Llinares, "Abstract convexity and fixed points," Journal of Mathematical Analysis and Applications, vol. 222, no. 1, pp. 138-150, 1998.

[3] J. Huang, "The matching theorems and coincidence theorems for generalized R-KKM mapping in topological spaces," Journal of Mathematical Analysis and Applications, vol. 312, no. 1, pp. 374-382, 2005.

[4] X. P. Ding, "Maximal element theorems in product FC-spaces and generalized games," Journal of Mathematical Analysis and Applications, vol. 305, no. 1, pp. 29-42, 2005.

[5] X. P. Ding, "Generalized KKM type theorems in FC-spaces with applications. I," Journal of Global Optimization, vol. 36, no. 4, pp. 581-596, 2006.

[6] S. Park, "Various subclasses of abstract convex spaces for the KKM theory," Proceedings of the National Institute of Mathematical Science, vol. 2, no. 4, pp. 35-47, 2007.

[7] B. Knaster, K. Kuratowski, and S. Mazurkiewicz, "Ein Beweis des fixpunktsatzes für $n$-dimensionale simplexe," Fundamenta Mathematicae, vol. 14, pp. 132-137, 1929.

[8] S. Park, "On generalizations of the KKM principle on abstract convex spaces," Nonlinear Analysis Forum, vol. 11, no. 1, pp. 67-77, 2006.

[9] S. Park, "Elements of the KKM theory on abstract convex spaces," Journal of the Korean Mathematical Society, vol. 45, no. 1, pp. 1-27, 2008.

[10] S. Park, "Fundamental theory of the KKM spaces," Fixed Point Theory and Applications. In press.

[11] S. Park, "Equilibrium existence theorems in KKM spaces," 2007, Nonlinear Analysis: Theory, Methods $\mathcal{E}$ Applications. In press.

[12] F.-C. Liu, “On a form of KKM principle and SupInfSup inequalities of von Neumann and of Ky Fan type," Journal of Mathematical Analysis and Applications, vol. 155, no. 2, pp. 420-436, 1991.

[13] M. Balaj, "Weakly G-KKM mappings, G-KKM property, and minimax inequalities," Journal of Mathematical Analysis and Applications, vol. 294, no. 1, pp. 237-245, 2004.

[14] G.-S. Tang, Q.-B. Zhang, and C.-Z. Cheng, "W-G-F-KKM mapping, intersection theorems and minimax inequalities in FC-space," Journal of Mathematical Analysis and Applications, vol. 334, no. 2, pp. 1481-1491, 2007.

[15] S. Park, "Examples of $\mathcal{K C}$-maps and $\mathcal{K O}$-maps on abstract convex spaces," Soochow Journal of Mathematics, vol. 33, no. 3, pp. 477-486, 2007.

[16] S. Park, "Coincidence, almost fixed point, and minimax theorems on generalized convex spaces," Journal of Nonlinear and Convex Analysis, vol. 4, no. 1, pp. 151-164, 2003.

[17] B. P. Sortan, Introduction to Axiomatic Theory of Convexity, Kishineff, New York, NY, USA, 1984.

[18] S. Park, "Ninety years of the Brouwer fixed point theorem," Vietnam Journal of Mathematics, vol. 27, no. 3, pp. 187-222, 1999.

[19] S. Park, "Elements of the KKM theory for generalized convex spaces," The Korean Journal of Computational \& Applied Mathematics, vol. 7, no. 1, pp. 1-28, 2000.

[20] S. Park, "Remarks on topologies of generalized convex spaces," Nonlinear Functional Analysis and Applications, vol. 5, no. 2, pp. 67-79, 2000.

[21] S. Park and W. Lee, "A unified approach to generalized KKM maps in generalized convex spaces," Journal of Nonlinear and Convex Analysis, vol. 2, no. 2, pp. 157-166, 2001.

[22] H. Komiya, "Convexity on a topological space," Fundamenta Mathematicae, vol. 111, no. 2, pp. 107-113, 1981.

[23] M. Lassonde, "On the use of KKM multifunctions in fixed point theory and related topics," Journal of Mathematical Analysis and Applications, vol. 97, no. 1, pp. 151-201, 1983.

[24] C. D. Horvath, "Contractibility and generalized convexity," Journal of Mathematical Analysis and Applications, vol. 156, no. 2, pp. 341-357, 1991.

[25] C. D. Horvath, "Extension and selection theorems in topological spaces with a generalized convexity structure," Annales de la Faculté des Sciences de Toulouse. Mathématiques, vol. 2, no. 2, pp. 253-269, 1993.

[26] X. P. Ding and T.M. Ding, "KKM type theorems and generalized vector equilibrium problems in noncompact FC-spaces," Journal of Mathematical Analysis and Applications, vol. 331, no. 2, pp. 1230-1245, 2006, (French).

[27] Q.-B. Zhang and C.-Z. Cheng, "Some fixed-point theorems and minimax inequalities in FC-space," Journal of Mathematical Analysis and Applications, vol. 328, no. 2, pp. 1369-1377, 2007. 


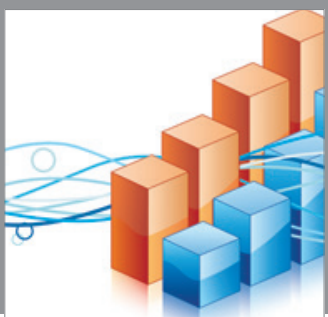

Advances in

Operations Research

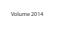

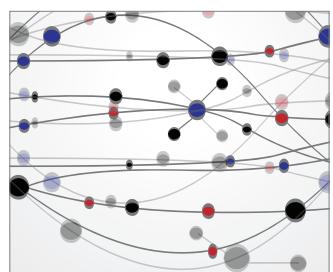

\section{The Scientific} World Journal
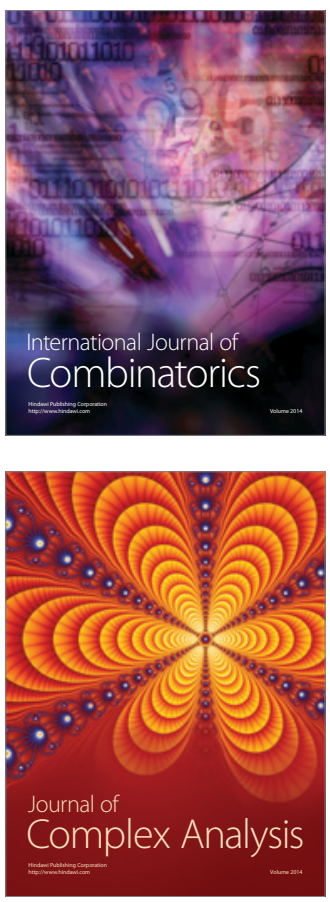

International Journal of

Mathematics and

Mathematical

Sciences
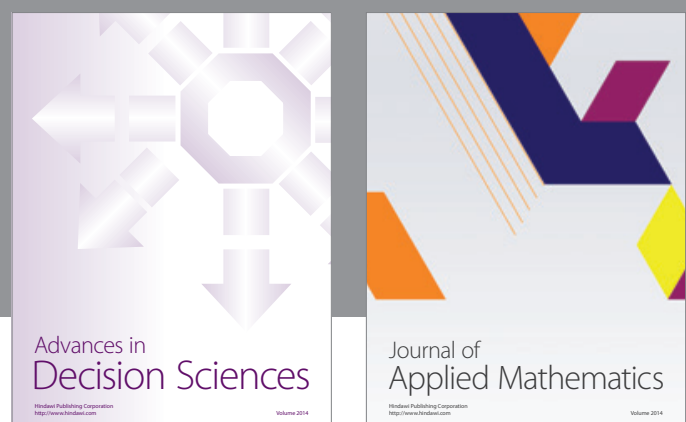

Journal of

Applied Mathematics
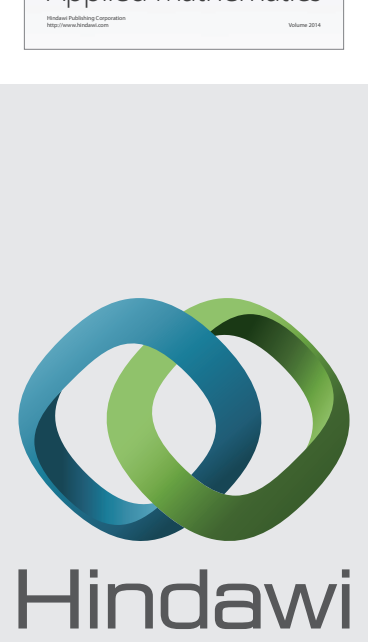

Submit your manuscripts at http://www.hindawi.com
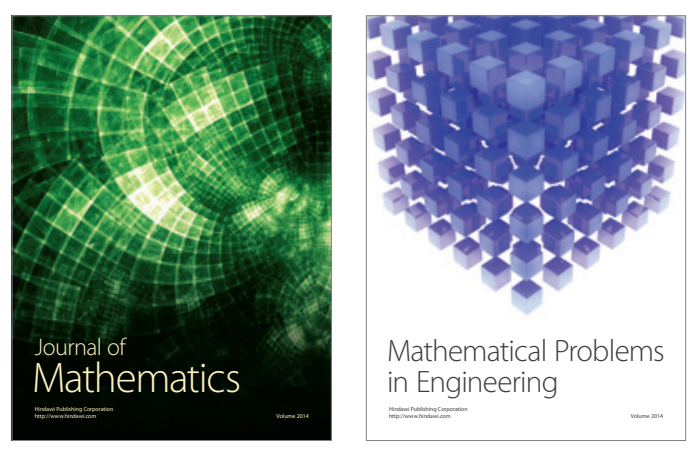

Mathematical Problems in Engineering
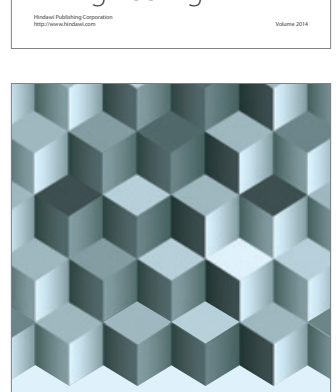

Journal of

Function Spaces
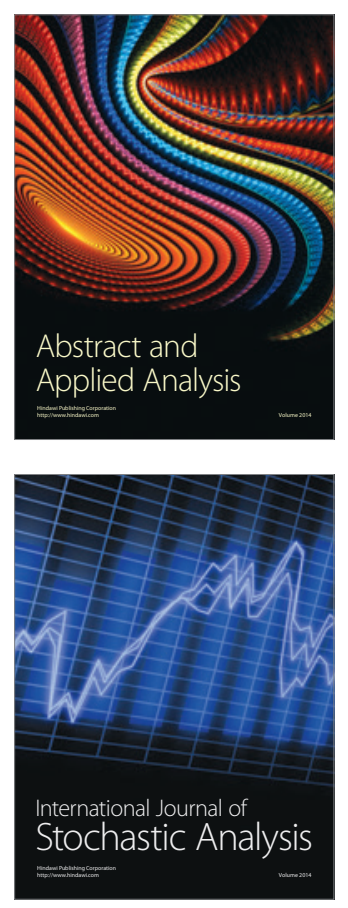

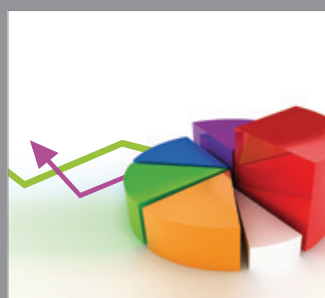

ournal of

Probability and Statistics

Promensencen
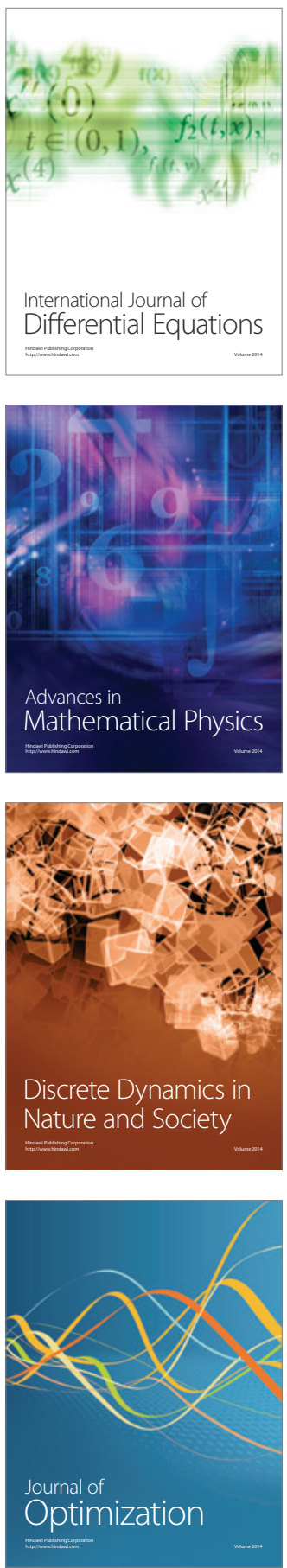